\title{
SneezeLove: Embodying Cultural Superstitions in Connected Devices
}

\author{
Malika Khurana \\ malikak@andrew.cmu.edu \\ School of Architecture, Carnegie Mellon University \\ Pittsburgh, Pennsylvania, USA \\ Daragh Byrne \\ daraghb@andrew.cmu.edu \\ School of Architecture, Carnegie Mellon University \\ Pittsburgh, Pennsylvania, USA
}

\author{
Zhenfang Chen \\ zhenfanc@andrew.cmu.edu \\ School of Architecture, Carnegie Mellon University \\ Pittsburgh, Pennsylvania, USA \\ Yang Bai \\ yangb@andrew.cmu.edu \\ School of Architecture, Carnegie Mellon University \\ Pittsburgh, Pennsylvania, USA
}

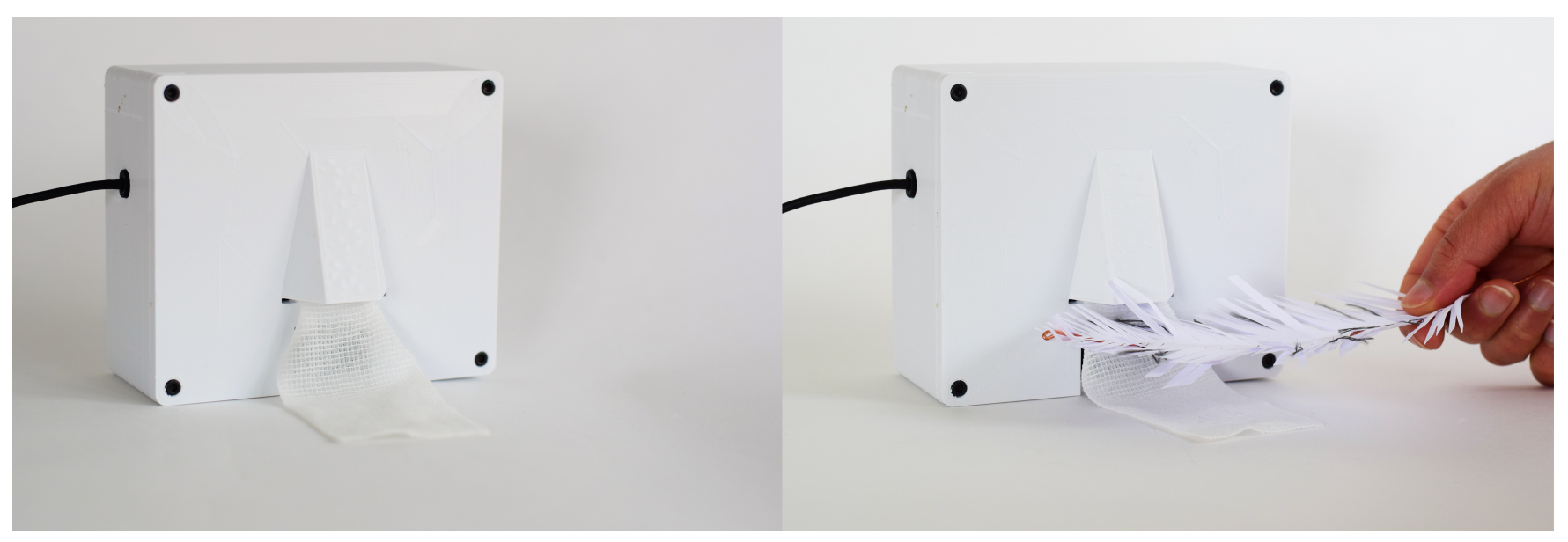

Figure 1: Prototype of SneezeLove

Left: the appearance of SneezeLove; Right: being triggered by a conductive feather

\begin{abstract}
Devices for remote communication and sending digital gestures have been widely explored in the field of ubiquitous computing. With the lack of social contact during the COVID-19 pandemic and renewed interest in these devices in mind, we suggest the affordances of a culturally-informed design approach. We present SneezeLove, a device that uses sneezes to communicate that someone is missing you. Users tickle the device's nose to send an electromechanical 'sneeze' to another device. The concept plays on a superstition in Chinese culture that attributes an additional layer of meaning to sneezes, and we found similar beliefs in other cultures that signify that someone out there is thinking of you: hiccups in India, "burning" ears in Ireland. The device, and its design approach, suggests how low-resolution messages can be enlivened and made
\end{abstract}

This work is licensed under a Creative Commons Attribution International 4.0 License.

DIS '21, fune 28-fuly 2, 2021, Virtual Event, USA

(C) 2021 Copyright held by the owner/author(s)

ACM ISBN 978-1-4503-8476-6/21/06

https://doi.org/10.1145/3461778.3462118 more personally meaningful by tapping into expressive and specific cultural superstitions.

\section{CCS CONCEPTS}

- Human-centered computing $\rightarrow$ Ubiquitous and mobile computing; Interaction design theory, concepts and paradigms; Human computer interaction (HCI).

\section{KEYWORDS}

Ubiquitous computing, cultural beliefs, superstitions, digital gestures, awareness, emotions, remote communication

\section{ACM Reference Format:}

Malika Khurana, Zhenfang Chen, Daragh Byrne, and Yang Bai. 2021. SneezeLove: Embodying Cultural Superstitions in Connected Devices. In Designing Interactive Systems Conference 2021 (DIS '21), June 28-fuly 2, 2021, Virtual Event, USA. ACM, New York, NY, USA, 5 pages. https://doi.org/10.1145/3461778. 3462118

\section{INTRODUCTION AND BACKGROUND}

Several decades have passed since the HCI community saw its first explorations of digital gesture communication [3, 4, 8-10, 20]. These tangible, networked objects support expressive interpersonal 
communication over distances, evoking "a visceral feeling of intimacy" that "conveys a direct sense of [the other's] corporeality" [9]. For example, touching a picture illuminates its counterpart, indicating you're thinking about that person [4]. Since then, many more projects have been added in a steady stream. With the current constraints on in-person socializing due to COVID-19, there is both renewed interest in this research direction [11] and a more urgent case to be made for imagining new and intimate ways of connecting people across distances. This moment also provides the opportunity to reflect on the history of gesture communication devices, and identify areas that are still largely unexplored.

Digital gesture communication devices build on ideas from a number of research areas in ubiquitous computing and the Internet of Things: information appliances [10], awareness technologies [9], ambient devices [17, 24], social objects [14], etc. Most gesture devices send a simple, often tangible, signal from one user to another remote user of an identical device, with the aim of creating an intimate and felt connection between pairs of people $[8,9,11,19]$. Though there is a wide variety of such projects, we find that few acknowledge cultural diversity in their designs, as they assume a Western context.

Other research in design and HCI has called attention to such cultural biases inherent in the design of technology products, namely that their assumed users are Americans living in a stereotypical and idealized home [1, 6, 15]. As Lucy Suchman observed in the 1990s, Silicon Valley has been regarded as the "here" where the future is defined for the rest of the world [21]. This US-centric perspective can be seen as a colonizing move that is harmful to the development and the identity of "non-centric" perspectives [12]. Recent work by Sultana and Ahmed illustrates how a postcolonial view of spirituality and local beliefs can enrich design and HCI [22]. Subsequent research has demonstrated that looking outside these assumptions provides enriching perspectives that can help "create spaces and technologies that people will want to use, not just admire from a distance" [1]. One approach to both close this distance and discover desirable alternatives has been to engage users in the design and creation of their devices. Audrey Desjardins' Alternative Avenues for IoT demonstrates how taking users' desires, unique environments, and identities as a starting point for IoT devices can open up new considerations of the values and beliefs embodied in our devices, and create more meaningful and positive relationships between humans and technology [6]. Further engaging users through a DIY approach, the Yo-Yo Machines project from the Interaction Research Studio at Goldsmiths empowers users to build their own gesture devices, personalize the materials and signals of their communication, and take pride in creating a tool for themselves [11]. This project also points to the seemingly universal desire in the pandemic to send tangible messages to people from whom we are now isolated.

Building on the history of gesture communication devices as well as recent critical reflections on technology, this short paper contributes this field in two ways: first we suggest an opportunity for culturally-informed communications that has largely not been explored and second we demonstrate this approach through material speculation [23] that yielded an interactive artifact, SneezeLove. This is next described.
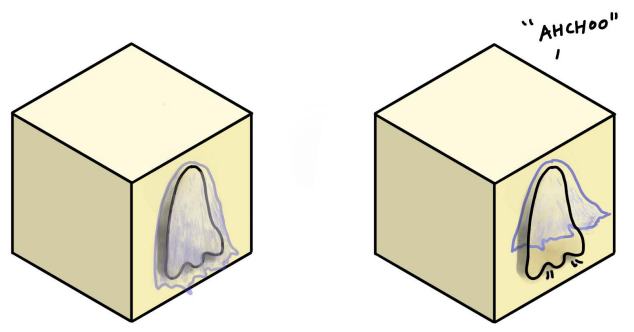

Figure 2: Conceptual sketch of SneezeLove

When the "nose" on one device is touched, the other connected device produces a "sneeze" to signify that someone is missing you.

\section{SNEEZELOVE}

Culture is the synthesis of our life experiences, values, and beliefs: invisible but constantly shaping how we understand and communicate meaning. In moments where we celebrate our shared culture with others, we feel a sense of belonging and an intimate connection with those people. These observations led us to believe that taking a user's cultural context and beliefs as inspiration for their device's affordances fosters interactions that are more personally meaningful and natural. Culturally-inspired affordances acknowledge the user's identity and utilize their prior associations with certain forms and gestures.

SneezeLove explores this potential of culturally-informed design by playing off a superstition in Chinese culture that says when you sneeze, someone is missing you. The device allows users to send and receive digital gestures in the form of a sneeze. By materializing this superstition as a metaphor for interaction, it creates an additional layer of intimacy between users: understanding the meaning of the sneeze relies on shared knowledge of the superstition. We grow up hearing such superstitions from our families and friends, learning the annotations to everyday occurrences that transform them into something special and noteworthy. Though they are generally not taken seriously, they allow us to find extra meaning, humor, and explanation in the mundane. We think of them as cultural inside jokes. Many cultures have superstitions specifically related to bodily phenomena, which lends itself to embodied interactions - both that are initiated by the user's body movement, and that are expressed through a tangible device "body." Related design research explorations include: leveraging animism for interactions with AI assistants [13], imagining alternative smart home devices informed by divination [16], and monsters and folklore as metaphors for machine processes [7]. We are not aware of work that has applied superstitions to tangible communication or digital gesture devices.

\section{MAKING SNEEZELOVE}

We approached our exploration of culturally-informed tangible communications as a process of material speculation [23] to imagine alternative ways to connect that are expressive and relate to the user's personal culture. Engaging our new everyday circumstance of remote living and the context of the pandemic as a resource for design, we operated as a distributed, remote team. We prepared an interactive device that reflected the conditions of being disconnected from homes, family and cultural contexts. The process of 


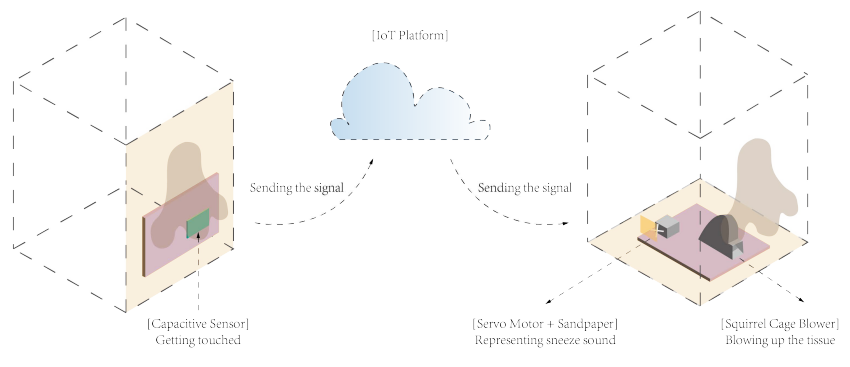

Figure 3: The technical approach and operation of SneezeLove

Two devices are connected via IoT platform; Once the capacitive sensor is touched, the servo motor and squirrel cage blower will work together to simulate a sneeze

creating SneezeLove can be divided into four stages: concept development, design, prototype implementation, and design evaluation.

\subsection{Concept Development}

Aware that significant work has already been done in this space, our aim was to find unexplored approaches to sending digital gestures and design interactions that would resonate in our current state of social isolation. We valued concepts that involve sharing ordinary but intimate moments, tangibly communicating presence through sound or motion, and identifying unexpected sites for design. Through research and speculation, we surfaced the observation that in one of the author's cultures, hiccups mean someone misses you. Further exploration foregrounded the range of specific beliefs that attribute social meaning to bodily phenomena that exist in a number of cultures around the world: sneezes in China, "burning" ears in Ireland, eye twitches in Trinidad [5]. We speculated how embodying the message of "I miss you" through a digital gesture grounded in one of these superstitions could create a playfully engaging and culturally meaningful interaction. As the majority of our social interactions in remote living are mediated by screens [18], the possibility of tangibly experiencing the presence of another person added additional value to unsettle the 'new normal.' We ultimately chose sneezing over the other superstitions as we felt it had the most evocative presence and offered potential to experiment with combined forms of feedback: air flow, movement, and sound.

\subsection{Design}

The SneezeLove device is enacted as a desktop or wall-mounted box with a nose protruding from its front surface, along with a feather made of conductive thread (see Figure 1). A tissue hangs below the nose and covers a small opening in the box, connected to a blower. To use SneezeLove, one user first sends a sneeze to signal that they are thinking of the other user. Using the conductive feather, they "tickle" under their device's "nose." A signal is sent via a cloud framework and received by another device. This triggers an electromechanical (and germ-free!) "sneeze" through a gust of air and a sneeze-like sound.

Early on, we decided on the literal form of a nose protruding from a box-like enclosure, to give the device a sense of humor and to directly acknowledge the playfulness of superstitions associated with bodily functions, with the thought that it would make its users smile when they sent or received a sneeze. The nose itself is abstracted and geometric, as we felt an overly realistic representation would both be discomforting and potentially so specific that it would become less relatable to its users (i.e. if it looked very different from their nose). We imagined the device would be placed at a fixed location in a user's home, and so a received sneeze should be felt even when the user is not close to the device. As we thought through the multi-sensory aspects of a sneeze that can be feasibly actuated and experienced at a distance, we chose to focus on the sound and gust of air. The gust of air gives the expression of release that is the essence of a sneeze, and it blows the tissue up as a visual, animated cue for user attention (see Figure 2). The sneeze sound serves as an ambient indicator that is subtle enough to not become distracting, much like we peripherally notice when someone actually in the same room as us sneezes [2]. Taking inspiration from foley artists, we experimented with generating an analog sound using materials rather than digital audio, as we believed it would give the sound effect a richer and more tangible presence, like the bellows of a cuckoo clock. To further push the embodied nature of the device, we aimed to make the act of sending the sneeze as materially engaged and playful as the sneeze itself. Again keeping in mind the sneeze metaphor as well as the sensors we could use to detect a trigger, we imagined the user could tickle the nose to trigger a sneeze on the paired device.

\subsection{Prototype Implementation}

The interaction we envisioned - tickling to trigger a sneeze, and a gust of air with a sound to represent the sneeze - was achieved by combining internet-connected microcontrollers, sensors and actuators, and foley-based material enactments. This is illustrated in Figure 3. To send a sneeze, a user brushes a feather containing conductive thread in the opening under the nose of one device, triggering a capacitive sensor. This device sends a signal to its partner device through the internet, triggering a sneeze. To actuate the sneeze, we utilized a squirrel cage blower for the gust of air, and a servo motor that rubs one piece of sandpaper against another to create a foley-inspired sneeze sound. This is performed just after the blower is triggered to generate a sneeze-like "ch" sound that appears in tandem with a gust of air. We originally explored noisegenerating materials that the blower could trigger directly, such as crinkling paper and blowing through a very narrow opening, but because of the limited strength of the squirrel case blower, we decided to incorporate the servo motor and sandpaper. The electronic components are all housed in an enclosure 3D printed in white PLA plastic. The enclosure contains brackets for mounting the electronics, and openings for access to wires and the capacitive sensor, as well as to attach the tissue that is actuated by the blower. This is illustrated in Figure 1.

\subsection{Design Evaluation}

Deploying the artifact for in-person user testing was not feasible at the time the project was completed, due to the COVID-19 pandemic and institutional restrictions. As a result, feedback from other users on the experience of living with the device could not be immediately 
gathered. Instead, our encounters with it, and feedback through design critiques from experts and students from a range of cultural backgrounds, has provided an initial opportunity for design evaluation. The superstition concept resonated with several participants in the critique, as they related the sneeze superstition to those they grew up hearing in their own cultures. Other feedback encouraged us to further explore the concept of disembodied communication, as a reference to the collective experience in the COVID-19 pandemic of relying on video and audio channels for communication. With so many of our social interactions happening virtually, we have grown poignantly aware of how these channels are lacking: they fail to capture subtleties of body language, presence, and intimacy of sharing space.

Through our own encounters with the prototype, we noted several ways it could be improved in the next iteration. As mentioned in section 3.1, a design goal was to evoke a playful and tangible presence, so the device enclosure could be finished to better utilize color and texture. The timing of the sneeze interaction could also be refined, and the sound of the sneeze amplified. The sneeze is a combination of sequential actions of a blower and a servo motor. Because the blower takes time to get to full speed and to stop spinning when turned off, even running it for the minimum time possible exceeds the duration of a sneeze. This detracts from the feeling of anticipation and release that we associate with sneezes, so we would like to find an actuator that can be triggered more instantaneously, and perhaps can move in two directions, as a slow inhale (anticipation) and sudden exhale (release). Because the "ch" sound of the sneeze is generated by the servo motor rubbing against a piece of sandpaper, the sound is fairly quiet and is at times obfuscated by the sound of the blower. We would like to keep the analog foley-inspired sounds in subsequent prototypes, rather than using digital sound, so we would need to explore a way of amplifying the sound.

\section{DISCUSSION AND FUTURE WORK}

Through designing SneezeLove, we found that the act of drawing from our real lived experiences and personal memories of culture allowed us to reflect on the empowerment that comes from creating devices that tap into personal meaning. We recognize that SneezeLove and the other concepts described are based on each of the authors' cultures, and may not relate to all users' personal cultures. Considering this, we wish to emphasize that they are examples serving to open a design space of culturally-informed technology devices. We are also sensitive to appropriation and that next steps should afford individual agencies and respectful materialization of any local superstitions and folk wisdoms. As such, our aim is to enable people to draw upon their own cultural beliefs and superstitions. Instead of purchasing devices with prescribed meanings and interactions, this would give agency to voice, materialize and involve individual beliefs, to reinforce their culture ascription and develop new personal associations with technology in the home.

As suggested by Yo-Yo Machines [11], this kind of customization can also stimulate playful imagination to enliven communication in this pandemic situation. Although these devices would have various appearances and perform differently, they still convey the same "small" message: "I miss you." Our work has identified many more opportunities for this variation: a number of cultures have beliefs associating mundane bodily functions with social meaning. This simultaneous universality and diversity is where the beauty of culture lies and the reason we cherish each culture.

Because of the limitations on user testing during the pandemic, we acknowledge that SneezeLove has not yet been formally deployed in different users' homes. We plan to evaluate the extent to which culturally-informed design helps create more personally meaningful digital gestures for users. By asking users to live with the device for at least one week, we hope they discover opportunities for unexpected moments of communication via SneezeLove. We aim to understand the nature of intimacy and meaning that is added to communication when it is in a medium that incorporates a "cultural inside joke" shared between its users. Do users feel closer when they communicate "I miss you" through an encoded medium rooted in shared superstitions?

\section{CONCLUSION}

This paper demonstrates how culture can meaningfully inform interaction affordances and the values that drive design. This builds on much prior work that calls attention to the normative, US-centric cultures of production in modern technology [1, 6, 21]. Through a process of material speculation, we designed and prototyped SneezeLove, a gesture communication device inspired by a Chinese superstition that says when you sneeze, it means someone is missing you. With SneezeLove, paired users can send the message "I miss you" through a sneeze, illustrating how communication mediums can made more intimate and immediate through tangibility and relevancy to the users' personal cultures. The familiarity and serendipity of such interactions has become precious during the COVID-19 pandemic, as people have been isolated from their loved ones and there have been few opportunities for happy chance encounters. As we imagine a post-pandemic world in which remote work has become more normalized, yet we have also become more acutely aware of the limitations of mainstream communication technologies, we believe the values behind SneezeLove will continue to be relevant. Our work emphasizes the importance of designs that are driven by alternative priorities, which put experiences, desirability, and emotional value first. We hope that this exploration further opens up superstitions as a rich proposition for culturally-informed communications and for continued exploration through design research.

\section{ACKNOWLEDGMENTS}

Thank you to David Chatting, Eddy Man Kim, and Dina El-Zanfaly for their time and generous feedback. This project was supported in part by funding from the Carnegie Mellon University FrankRatchye Fund For Art @ the Frontier.

\section{REFERENCES}

[1] Genevieve Bell and Joseph Kaye. 2002. Designing Technology for Domestic Spaces: A Kitchen Manifesto. Gastronomica 2, 2 (2002), 46-62. https://doi.org/ 10.1525/GFC.2002.2.2.46

[2] A. Case. 2015. Calm Technology: Principles and Patterns for Non-Intrusive Design. O'Reilly Media. https://books.google.com/books?id=Mp88CwAAQBAJ

[3] Angela Chang and Hiroshi Ishii. 2006. Sensorial Interfaces. In Proceedings of the 6th Conference on Designing Interactive Systems (University Park, PA, USA) 
(DIS '06). Association for Computing Machinery, New York, NY, USA, 50-59. https://doi.org/10.1145/1142405.1142415

[4] A. Chang, Ben Resner, Brad Koerner, XingChen Wang, and H. Ishii. 2001. LumiTouch: an emotional communication device. CHI '01 Extended Abstracts on Human Factors in Computing Systems (2001). https://doi.org/10.1145/634067.634252

[5] Krystal D'Costa. 2011. Anatomy of a Superstition: When Your Eye "Jumps". https://blogs.scientificamerican.com/anthropology-in-practice/ anatomy-of-a-superstition-when-your-eye-jumps/

[6] Audrey Desjardins, Jeremy E. Viny, Cayla Key, and Nouela Johnston. 2019. Alter native Avenues for IoT: Designing with Non-Stereotypical Homes. In Proceedings of the 2019 CHI Conference on Human Factors in Computing Systems (Glasgow, Scotland Uk) (CHI '19). Association for Computing Machinery, New York, NY, USA, 1-13. https://doi.org/10.1145/3290605.3300581

[7] Graham Dove and Anne-Laure Fayard. 2020. Monsters, metaphors, and machine learning. In Proceedings of the 2020 CHI Conference on Human Factors in Computing Systems. 1-17. https://doi.org/10.1145/3313831.3376275

[8] A Dunne and F Raby. 1995. Fields and Thresholds, Architects in Cyberspace. Architectural Design, Neil Spiller (ed) 118 (1995), p64.

[9] Bill Gaver. 2002. Provocative Awareness. Computer Supported Cooperative Work (CSCW) 11, 3-4 (Sept. 2002), 475-493. https://doi.org/10.1023/A:1021277326673

[10] Bill Gaver and Heather Martin. 2000. Alternatives: exploring information appliances through conceptual design proposals. In Proceedings of the SIGCHI conference on Human factors in computing systems. 209-216. https://doi.org/10.1145/ 332040.332433

[11] William Gaver, Andy Boucher, Dean Brown, David Chatting, Naho Matsuda, Liliana Ovalle, Andy Sheen, and Michail Vanis. 2020. Yo Yo Machines. https: //www.yoyomachines.io/

[12] Lilly Irani, Janet Vertesi, Paul Dourish, Kavita Philip, and Rebecca E Grinter 2010. Postcolonial computing: a lens on design and development. In Proceedings of the SIGCHI conference on human factors in computing systems. 1311-1320. https://doi.org/10.1145/1753326.1753522

[13] Betti Marenko and Philip Van Allen. 2016. Animistic design: how to reimagine digital interaction between the human and the nonhuman. Digital Creativity 27, 1 (2016), 52-70. https://doi.org/10.1080/14626268.2016.1145127

[14] Bjorn Nansen, Luke van Ryn, Frank Vetere, Toni Robertson, Margot Brereton, and Paul Dourish. 2014. An internet of social things. In Proceedings of the 26th Australian Computer-Human Interaction Conference on Designing Futures: the Future of Design. 87-96. https://doi.org/10.1145/2686612.2686624
[15] Doenja Oogjes, William Odom, and Pete Fung. 2018. Designing for an Other Home: Expanding and Speculating on Different Forms of Domestic Life. In Proceedings of the 2018 Designing Interactive Systems Conference (Hong Kong, China) (DIS '18). Association for Computing Machinery, New York, NY, USA, 313-326. https://doi.org/10.1145/3196709.3196810

[16] James Pierce and Carl DiSalvo. 2017. Dark Clouds, Io\&\#!+, and [Crystal Ball Emoji] Projecting Network Anxieties with Alternative Design Metaphors. In Proceedings of the 2017 Conference on Designing Interactive Systems. 1383-1393. https://doi.org/10.1145/3064663.3064795

[17] Zachary Pousman and John Stasko. 2006. A Taxonomy of Ambient Information Systems: Four Patterns of Design. In Proceedings of the Working Conference on Advanced Visual Interfaces (Venezia, Italy) (AVI '06). Association for Computing Machinery, New York, NY, USA, 67-74. https://doi.org/10.1145/1133265.1133277

[18] Yvonne Rogers. 2020. Is remote the new normal? reflections on Covid-19, technology, and humankind. Interactions 27, 4 (2020), 42-46. https://doi.org/10.1145/ 3403586

[19] Hooman Aghaebrahimi Samani, Rahul Parsani, Lenis Tejada Rodriguez, Elham Saadatian, Kumudu Harshadeva Dissanayake, and Adrian David Cheok. 2012. Kissenger: design of a kiss transmission device. In Proceedings of the Designing Interactive Systems Conference on - DIS '12 (Newcastle Upon Tyne, United Kingdom). ACM Press, 48. https://doi.org/10.1145/2317956.2317965

[20] R. Strong and William W. Gaver. 1996. Feather, Scent, and Shaker: Supporting Simple Intimacy.

[21] L. Suchman. 2011. Anthropological Relocations and the Limits of Design. Annual Review of Anthropology 40 (2011), 1-18. https://doi.org/10.1146/ANNUREV. ANTHRO.041608.105640

[22] Sharifa Sultana and Syed Ishtiaque Ahmed. 2019. Witchcraft and HCI: Morality, Modernity, and Postcolonial Computing in Rural Bangladesh. In Proceedings of the 2019 CHI Conference on Human Factors in Computing Systems (Glasgow, Scotland Uk) (CHI '19). Association for Computing Machinery, New York, NY, USA, 1-15. https://doi.org/10.1145/3290605.3300586

[23] Ron Wakkary, William Odom, Sabrina Hauser, Garnet Hertz, and Henry Lin. 2015. Material speculation: Actual artifacts for critical inquiry. In Proceedings of The Fifth Decennial Aarhus Conference on Critical Alternatives. 97-108. https: //doi.org/10.7146/AAHCC.V1I1.21299

[24] Mark Weiser and John Seely Brown. 1997. The coming age of calm technology. In Beyond calculation. Springer, 75-85. https://doi.org/10.1007/978-1-4612-0685-9_6 